Full length article

\title{
Effect of powder characteristics and oxygen content on modifications to the microstructural topology during hot isostatic pressing of an austenitic steel
}

\author{
S. Irukuvarghula ${ }^{\text {a, }}{ }^{*}$, H. Hassanin $^{\text {b }}$, C. Cayron ${ }^{\text {c }}$, M. Aristizabal ${ }^{\text {d }}$, M.M. Attallah ${ }^{\text {e, }}$ \\ M. Preuss ${ }^{\text {a }}$ \\ a School of Materials, University of Manchester, M1 3BB, UK \\ ${ }^{\mathrm{b}}$ Department of Civil Engineering and Industrial Design, University of Liverpool, Liverpool, L69 3BX, UK \\ ${ }^{\mathrm{c}}$ Ecole Polytechnique Fedéralé de Lausanne (EPFL), Rue de la Maladière 71b, 2000, Neuchâtel, Switzerland \\ d Ceit-IK4, PO Manuel Lardizabal 15, 20018, Donostia-San Sebastian, Spain \\ e School of Metallurgy and Materials, University of Birmingham, Edgbaston, B15 2TT, UK
}

\section{A R T I C L E I N F O}

\section{Article history:}

Received 27 November 2018

Received in revised form

30 March 2019

Accepted 31 March 2019

Available online 5 April 2019

\section{Keywords:}

Austenitic steels

Powder metallurgy

Hot isostatic pressing

Recrystallization

Twin related domains

\begin{abstract}
A B S T R A C T
The effect of powder size distribution and oxygen content on the extent of multiple twinning and spatial distribution of oxide inclusions in hot isostatic pressed (HIPed) 316L steels was investigated using powders with different characteristics. Modifications to, and differences in their microstructural topology, were tracked quantitatively by evaluating the metrics related to twin related domains (TRDs) on specimens produced by interrupting the HIPing process at various points in time. Results revealed that powder size distribution has a strong effect on the extent of multiple twinning in the fully HIPed microstructure, with specimens produced using narrow distribution showing better statistics (i.e., homogeneously recrystallized) than the ones produced using broad size distribution. The oxide inclusion density in fully HIPed microstructures increased with the amount of oxygen content in the powders while prior particle boundaries (PPBs) were only observed in the specimens that were HIPed using broad powder distribution. More importantly, results clearly revealed that the spatial distribution of the inclusions was strongly affected by the homogeneity of recrystallization. Implications of the results are further discussed in a broader context, emphasizing the importance of utilizing the occurrence of solid state phase transformations during HIPing for controlling the microstructure evolution.
\end{abstract}

๑ 2019 Acta Materialia Inc. Published by Elsevier Ltd. This is an open access article under the CC BY license (http://creativecommons.org/licenses/by/4.0/).

\section{Introduction}

Powder hot isostatic pressing (HIPing) is a manufacturing process that is used to produce near net shape components with fine grain size, chemical homogeneity, and improved inspectability [1]. Used in many industries, including oil \& gas and aerospace, it is a thermomechanical process in which powder encapsulated in a canister is consolidated to theoretical density by the application of temperature and pressure. The HIPing conditions, chosen according to the material (see for e.g., Table 1 in Ref. [1]), ensure complete densification of the powder compact by the end of the HIPing cycle. While the densification kinetics during HIPing for various alloys

\footnotetext{
* Corresponding author.

E-mail address: sandeep.irukuvarghula@twi.co.uk (S. Irukuvarghula).
}

have been studied to considerable detail [2-7], the effect of powder characteristics on the microstructure development during HIPing, and on fully HIPed microstructure, still remains unclear, and is of significant interest.

Albeit the applied pressure during HIPing is isostatic, powders deform inhomogeneously depending on their size; i.e., small particles deform more than large particles [5,6,8-11]. In powder HIPed 316L austenitic stainless steels, it has previously been demonstrated that deformation of the powder is a prerequisite for recrystallization to occur during HIPing [11]. This has been linked to the absence of annealing twins ( $\Sigma 3$ boundaries in coincidence site lattice formalism) and associated twin chains in powder particles that do not undergo plastic deformation; these non-deformed powders can be discerned by their prior particle boundaries (PPBs) in the fully consolidated microstructure [11]. This observation has a more general applicability to low-medium stacking fault 
energy (SFE) materials processed by powder HIPing, and is not limited to 316L steels (see for e.g. Refs. [12,13], for microstructures of powder HIPed Inconel 718).

It is known that inclusions and second phase particles act as void nucleation sites during ductile fracture, and strongly affect material toughness [14-16]. Compared to conventionally processed materials, powders contain an order of magnitude more oxygen, which, depending on its solubility in the material, can be in the form of oxide inclusions (or oxycarbides, depending on the chemistry of the alloy) in the microstructure of a fully HIPed component. It has previously been demonstrated that oxide inclusions lower the impact toughness of powder HIPed 316L and 304L steels [17,18]. In powder HIPed nickel-base superalloys, presence of inclusions at PPBs in the as-HIPed and heat treated state has been linked to the reduction in their ductility and stress rupture properties [12,13,19], although post-HIP hot working has been shown to "break" the PPB networks and randomize the spatial distribution of inclusions [20-24]. In other words, as a result of recrystallization during postHIP thermomechanical processing, inclusions at PPBs are pushed by moving grain boundaries, and therefore, are homogeneously distributed in the bulk. More specifically, spatial redistribution of the inclusions, which are initially present at PPBs, is promoted by the formation and propagation of twin chains (i.e., recrystallization) during hot working.

Since the microstructure in low-medium SFE materials evolves by recrystallization during HIPing (provided there is enough stored energy from particle deformation) [11], understanding the effect of powder characteristics on microstructure development, and more specifically, on the extent and homogeneity of recrystallization and its effect on the spatial distribution of inclusions, will give insights for the development of high value manufacturing of near net shape HIPed components (e.g., Nickel-base superalloys and austenitic steels for aerospace, oil \& gas, and nuclear industries). Therefore, in the present study, by HIPing 316L powders (a representative low-medium SFE material) with different size distributions and oxygen content, we aim to address (a) the effect of powder size distribution on recrystallization and the extent of multiple twinning during HIPing (b) the effect of oxygen content in the powders on the inclusion size and density in the fully HIPed microstructure and (c) the effect of recrystallization on the spatial distribution of oxide inclusions during HIPing.

Modifications to the microstructural topology of different powders during HIPing were investigated by quantifying multiple twinning using the metrics related to twin related domains (TRDs). The notion of TRDs as a characteristic microstructural dimension in low-medium SFE materials that are prone to recrystallization was first proposed by Gertsman and Henager [25], and later developed by Reed et al. [26,27] and Cayron [28,29]. Specifically, TRDs represent clusters of grains that are related by $\Sigma 3^{n}$ misorientations, and connected by chains of $\Sigma 3$ boundaries [25]. Therefore, the entire recrystallized microstructure consists of multiple twinned clusters, i.e., TRDs. Since $\Sigma 3$ boundaries are generally immune to percolation phenomena (such as crack propagation), and because all grains within TRDs are connected by $\Sigma 3$ boundaries, it was suggested that the material performance is controlled at the length scale of TRDs [25]. Reed et al. [30] and Barr et al. [31], by providing experimental evidence, clearly demonstrated the existence of a correlation between TRD size and material response towards intergranular phenomena.

Therefore, in this study, data analysis was focused towards identifying TRDs and quantifying multiple twinning. Using the metrics related to TRDs, we analyze the data from microstructures produced by interrupting the HIP process at various points in time. Inclusion density (i.e., \#/unit area) is measured in the fully HIPed microstructures and is linked back to the oxygen content in the as- received powders. Connections between powder size distribution, PPBs, oxygen content, and the spatial distribution of inclusions on recrystallization are made, and are shown to be associated with the powder deformation during HIPing. Suggestions in terms of powder size distribution and oxygen content are put forward, that produce homogeneously recrystallized microstructures with low volume fraction and random spatial distribution of inclusions (i.e., not on PPBs) in a near net shape component, potentially eliminating the need for post-HIP thermomechanical processing.

\section{Materials and methods}

\subsection{Experimental}

Six powders of 316L austenitic steel with different characteristics (chemical and physical) were used in this study. ${ }^{1}$ Specifically, four nitrogen atomised powders were obtained from commercial vendors (designated as C, D, E, and F) while two powders were gas atomised at TLS technik, Germany (atomised using argon and nitrogen; designated as $A$ and $B$, respectively), from a forged plate supplied by Rolls-Royce. Particle size distribution of the six powders is shown in Fig. 1. It can be seen that powders $C$ and D have much narrower size distribution while others have much broader distribution. Statistical quantities such as $\mathrm{D}_{10}, \mathrm{D}_{50}$, $\mathrm{D}_{90}$, median, and mean for all particle size distributions are given in Table 1 while their chemical composition and some of their characteristics are shown in Tables 2 and 3, respectively. The oxygen content in the powders varies from $110 \mathrm{ppm}$ to $610 \mathrm{ppm}$ and the tap density from 4.9 to $5.7 \mathrm{~g} \mathrm{~cm}^{-3}$. For interrupted HIP runs, powders were first filled in $25 \mathrm{~mm}$ diameter, $30 \mathrm{~mm}$ height, and $2 \mathrm{~mm}$ thick mild steel canisters, vibrated and vacuum degassed at room temperature before hot crimping the evacuation tube. The canisters were then HIPed using simultaneous application of temperature and pressure, at $950^{\circ} \mathrm{C}, 1000^{\circ} \mathrm{C}, 1050^{\circ} \mathrm{C}, 1120^{\circ} \mathrm{C}$, without any dwell time, at $103 \mathrm{MPa}$.

Additionally, powders were filled in mild steel canisters with $76 \mathrm{~mm}$ outside diameter, $2.3 \mathrm{~mm}$ wall thickness, $200 \mathrm{~mm}$ height and HIPed at $1160{ }^{\circ} \mathrm{C}$ and $103 \mathrm{MPa}$, and held at those conditions for $4 \mathrm{~h}$. The canisters were then solution annealed at $1050^{\circ} \mathrm{C}$ for $1.5 \mathrm{~h}$

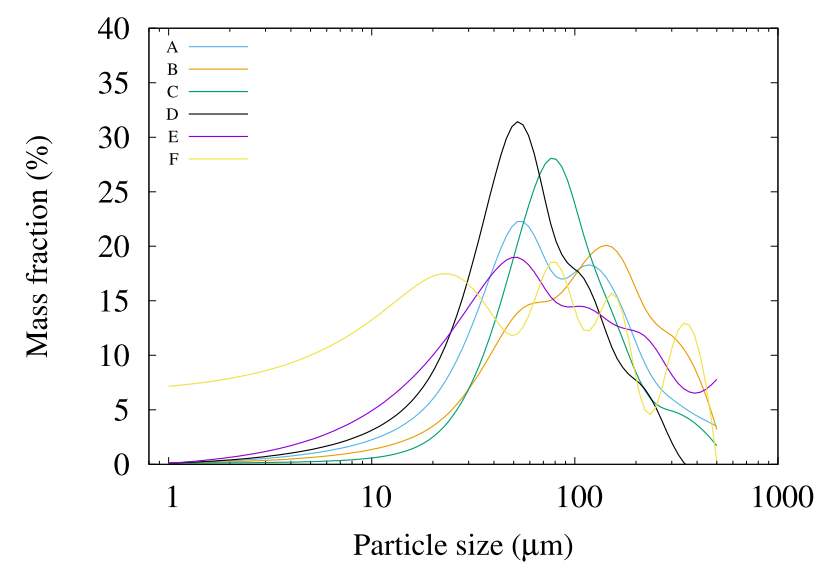

Fig. 1. Particle size distribution of various powders.

\footnotetext{
${ }^{1}$ Data for one of the six powders (powder C) were presented in our earlier study [11], and we show them here along with the data of five powders to demonstrate better, the correlation between powder characteristics and their microstructure evolution during HIP consolidation.
} 
Table 1

Statistical quantities associated with the particle size distributions.

\begin{tabular}{lllllll}
\hline Metric $(\mu \mathrm{m})$ & A & B & C & D & E & F \\
\hline $\mathrm{D}_{10}$ & 25 & 34 & 32 & 21 & 20 & 12 \\
$\mathrm{D}_{50}$ & 82 & 106 & 76 & 61 & 87 & 146 \\
$\mathrm{D}_{90}$ & 205 & 249 & 184 & 147 & 276 & 153 \\
Median & 82 & 106 & 76 & 61 & 87 & 146 \\
Mean & 126 & 151 & 115 & 91 & 147 & 106 \\
\hline
\end{tabular}

and water quenched. These are designated as fully HIPed samples (or fully consolidated state) in this study. For microstructural examination, specimens were sectioned, ground and polished using standard metallographic procedures. Final polishing was performed on a vibratory polisher using colloidal silica. Backscattered electron (BSE), secondary electron (SE) images, energy dispersive spectroscopy (EDS) data for chemical mapping, and Electron Backscatter Diffraction (EBSD) data were acquired on a Field Emission Gun Scanning Electron Microscope. EBSD maps were acquired to study the differences in the evolution of grain boundary network in all specimens during the HIPing process. A step size of $0.5 \mu \mathrm{m}$ and $1 \mu \mathrm{m}$ were used for partially HIPed and fully HIPed specimens, respectively. For statistical analysis of the data, EBSD maps from 5 random locations were acquired.

\subsection{Data analysis}

Differences in the microstructural topology of the fully HIPed specimens were initially studied by quantifying the distribution of the triple junctions (TJs) associated with $\Sigma 3$ boundaries. Specifically, three types of TJs were identified: $J_{0}$ type triple junction containing three boundaries which are not $\Sigma 3, J_{1}$ type containing one $\Sigma 3$ boundary, and $J_{2}$ type containing two $\Sigma 3$ boundaries. TJs were identified using scripts written in MATLAB, and executed as part of the freely available MTEX package [32]. This approach allows qualitative understanding of the extent of twinning. For the EBSD data analysis using MTEX, grains were reconstructed with a threshold misorientation of $5^{\circ}$. A tolerance angle of $3^{\circ}$ from the exact misorientation was used for identifying $\Sigma 3$ boundaries. Nonindexed pixels, which were typically less than $1.5 \%$ of the total pixels in the data (hit-rate in most cases was around 99\%), were assigned to surrounding grains. Identification of higher order twins, the evolution of TRDs and the associated metrics for intermediate HIP states for all specimens was performed using ARPGE software developed by Cayron [33]. More specifically, average values of size of TRDs, number of grains per TRD $\left(\left\langle N_{g}\right\rangle\right)$, length of longest chain $(\langle L L C\rangle)$, polysynthetism $(\langle p\rangle)$, and twinning anisotropy $(\langle a\rangle)$ were analyzed to better quantify multiple twinning.

Detailed description and their interpretation are discussed in Ref. [29]. Briefly, length of longest chain and polysynthetism represent the longest chain of $\Sigma 3$ twins in each grain and the tendency to form twin chains of type A-B-A-B-A ... where the grains $A$ and $B$ are linked by $\Sigma 3$ boundaries in a TRD, respectively. Twinning anisotropy, which depends on the choice of the primary
Table 3

Physical properties/characteristics of the powders.

\begin{tabular}{lllllll}
\hline Property & A & B & C & D & E & F \\
\hline Apparent density $\left(\right.$ g.cm $\left.^{-3}\right)$ & 5.4 & 5.3 & 4.5 & 4.9 & 5.2 & 5.1 \\
Tap density $\left(\right.$ g.cm $\left.^{-3}\right)$ & 5.7 & 5.5 & 4.9 & 5.2 & 5.5 & 5.6 \\
Flowability (FFC) & 17 & 23 & 10 & 19 & 18 & 16 \\
Satellites & Yes & Yes & Yes & Yes & Yes & Yes \\
\hline
\end{tabular}

grain used for reconstructing the TRD, represents the structure of the TRD. In other words, it describes the morphology of the TRD from its twinning tree. For statistical analysis of the oxide inclusions, several BSE images of the specimens were acquired and processed using imageJ software [34]. The average size of inclusions, their number density (\#/unit area), and the nearest neighbour distance were computed with built-in plugins available in imageJ.

\section{Results}

Fig. 2 shows representative images highlighting general microstructural aspects of as-received powder. Fig. 2a shows morphology of the powder; it is seen that the powder particles are spherical and contain smaller particles (referred to as satellites) on their surfaces. Such a morphology was observed for all powders, with powder $C$ having the most number of satellites. Presence of satellites on powder surfaces reduces flowability and affects powder packing. Fig. $2 \mathrm{~b}$ highlights the surface features of the powder. Small particles, $<1 \mu \mathrm{m}$, are seen to adhere the powder surface, presumably from the gas-atomization process. Fig. 2c shows the SE image along with the chemical maps of nickel and molybdenum obtained on polished surface of a powder particle. Nickel, and to a lesser extent, molybdenum, are seen to be segregated in what appears to be a cellular microstructure. Fig. $2 \mathrm{~d}$ shows the grain boundary misorientations of a particle from the as-received powder. The misorientations are colour coded according to the scheme proposed by Patala et al. [35,36], which allows complete misorientation representation (axis and angle) of grain boundaries. It is seen that the boundaries have a jagged appearance and are predominantly high angle.

While the grain boundaries in as-received powder principally are random high angle, the fully HIPed specimens on the other hand contain a large fraction of annealing twins (Fig. 3), indicating that the powders undergo recrystallization during consolidation by HIPing. Qualitatively, Fig. 3 also reveals differences in the microstructures of specimens HIPed with different powders. Specifically, it can be seen that the specimens consolidated using powders A, B, and $\mathrm{F}$ show an inhomogeneously recrystallized microstructure with large grains surrounded by several small recrystallized grains (Fig. 3a, b, and f, respectively) while the specimens HIPed using powders $\mathrm{C}, \mathrm{D}$, and $\mathrm{E}$ are more homogeneously recrystallized (Fig. 3c, d, and Fig. 3e, respectively).

Differences in their microstructures were evaluated first by analyzing the distribution of $J_{0}, J_{1}$, and $J_{2}$ type triple junctions. Fig. 4

Table 2

Chemical composition (in wt\%) of 316L stainless steel powders determined using inductively coupled plasma mass spectrometry and intert gas fusion.

\begin{tabular}{|c|c|c|c|c|c|c|c|c|c|c|c|}
\hline Powder & $\mathrm{Cr}$ & $\mathrm{Mn}$ & Mo & $\mathrm{Ni}$ & $\mathrm{P}$ & $\mathrm{Si}$ & $\mathrm{C}$ & $\mathrm{S}$ & $\mathrm{N}$ & 0 & $\mathrm{Fe}$ \\
\hline A & 17.93 & 1.83 & 2.10 & 10.09 & 0.036 & 0.35 & 0.028 & 0.001 & 0.079 & 0.029 & Bal. \\
\hline B & 17.84 & 1.85 & 2.08 & 10.07 & 0.035 & 0.39 & 0.029 & 0.001 & 0.129 & 0.011 & Bal. \\
\hline C & 16.44 & 1.32 & 2.08 & 10.14 & 0.023 & 0.57 & 0.018 & 0.002 & 0.098 & 0.021 & Bal. \\
\hline D & 16.94 & 1.48 & 2.41 & 10.45 & 0.017 & 0.69 & 0.016 & 0.007 & 0.118 & 0.061 & Bal. \\
\hline E & 17.9 & 1.84 & 2.44 & 11.78 & 0.009 & 0.73 & 0.02 & 0.007 & 0.061 & 0.022 & Bal. \\
\hline $\mathrm{F}$ & 17.6 & 0.64 & 2.2 & 11.3 & 0.012 & 0.69 & 0.012 & 0.010 & 0.05 & 0.0149 & Bal. \\
\hline
\end{tabular}




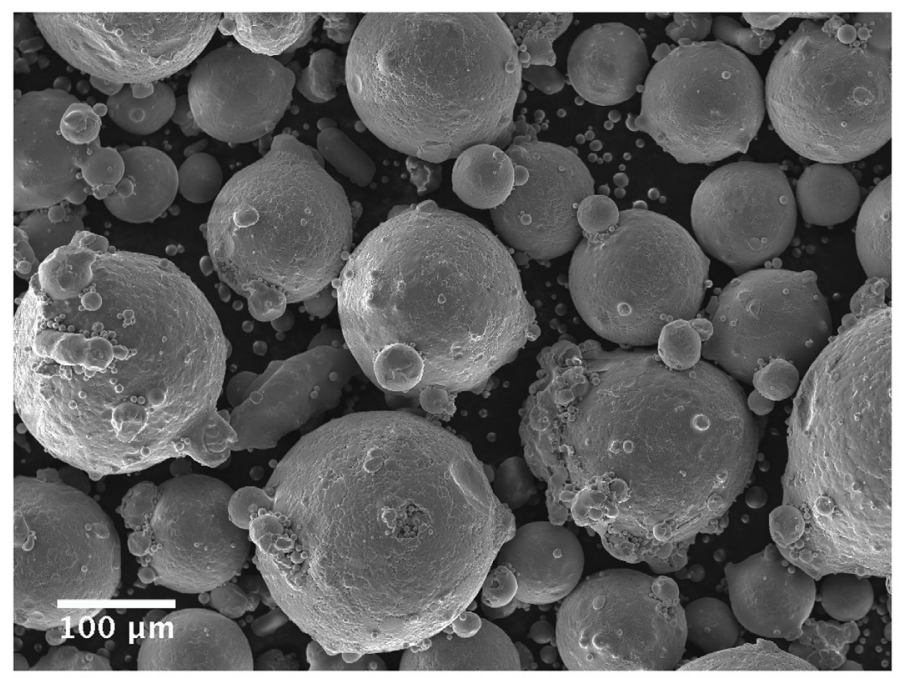

(a)

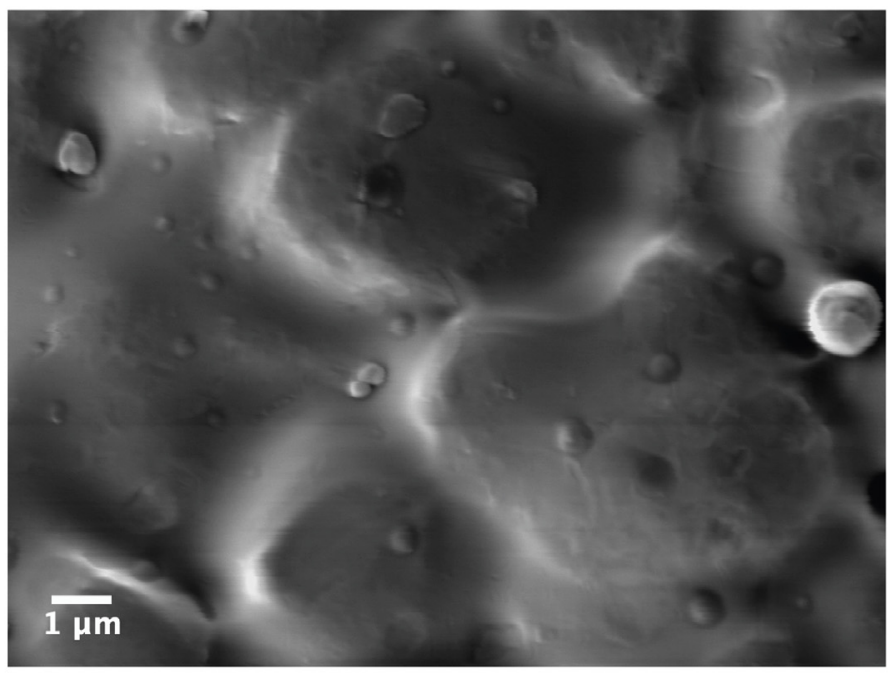

(b)
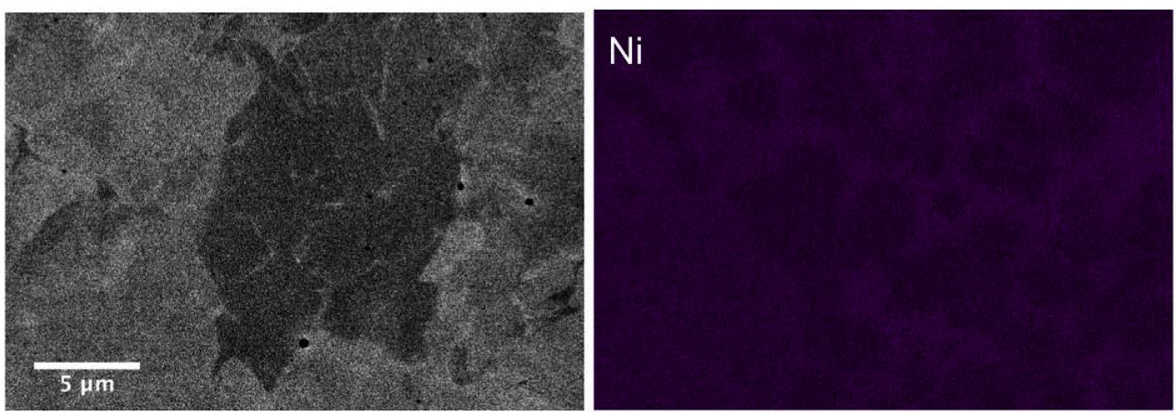

(c)
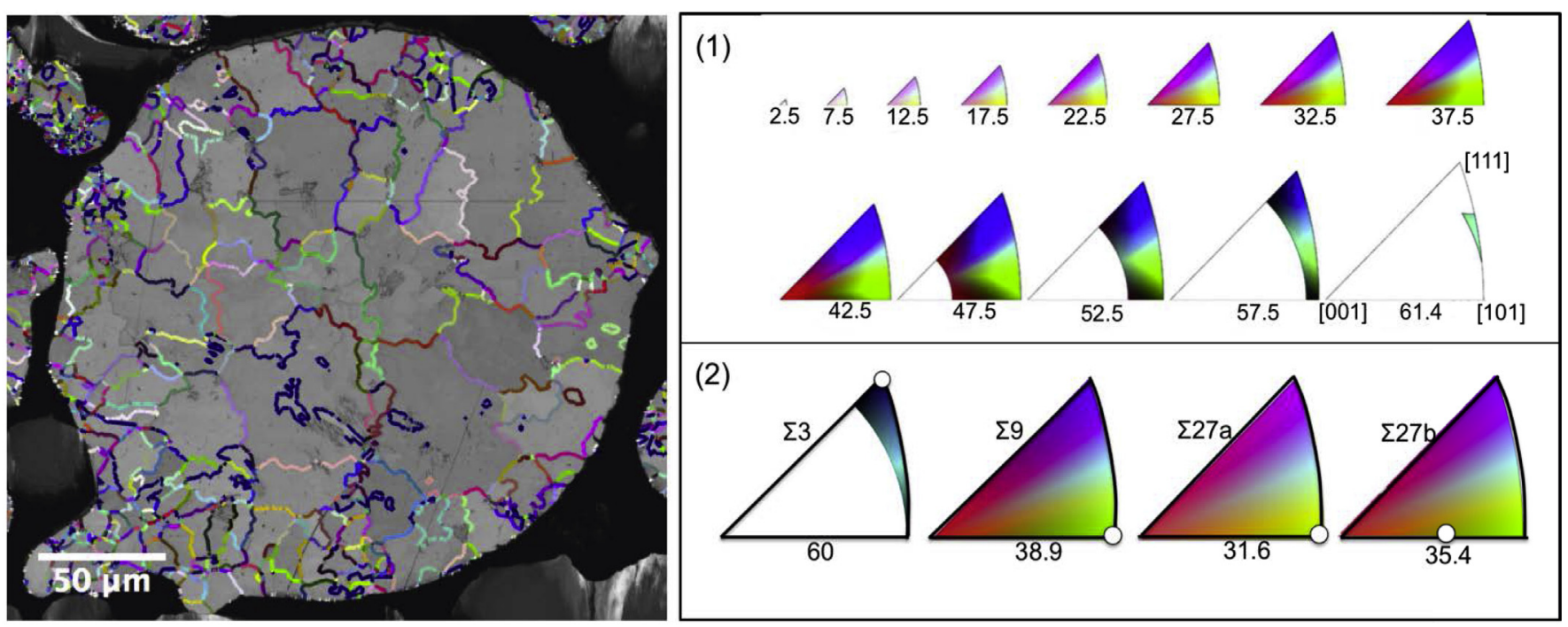

(d)

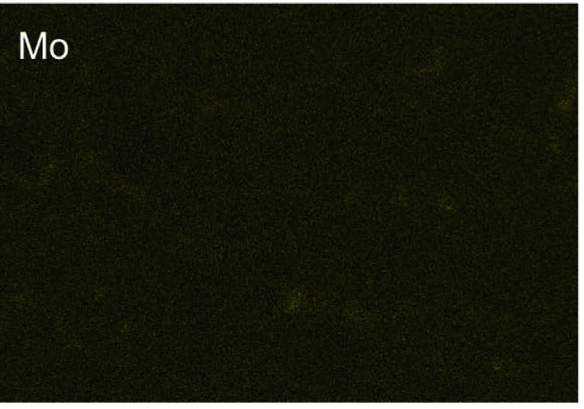




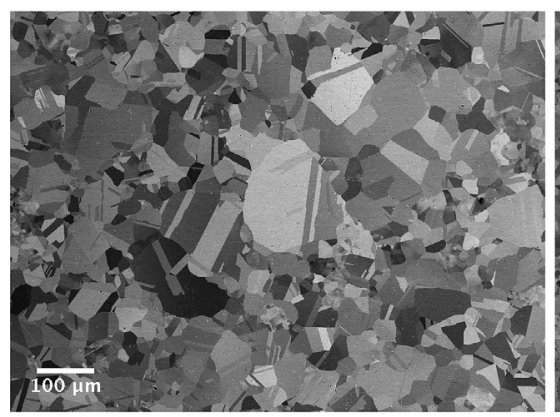

(a)

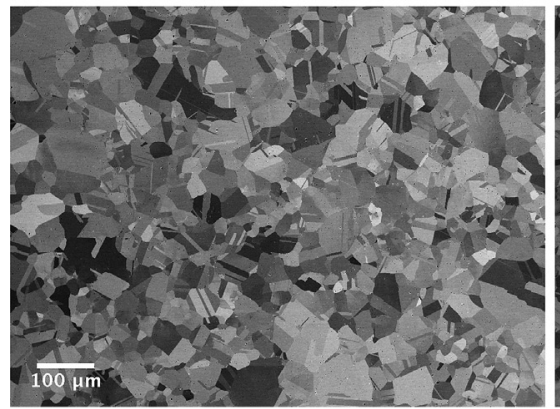

(d)

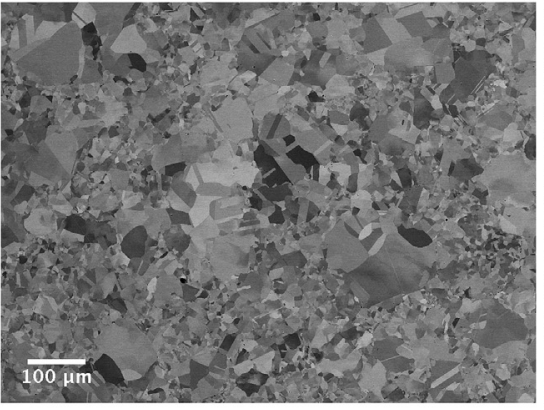

(b)

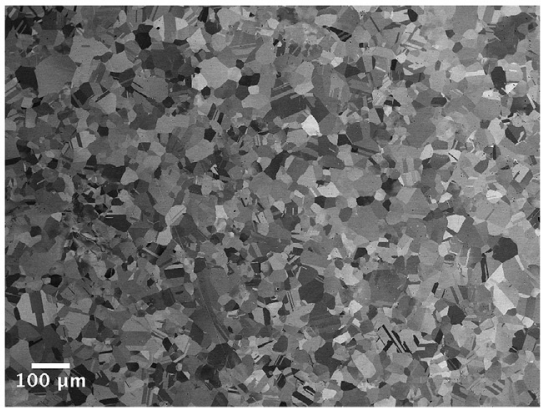

(e)

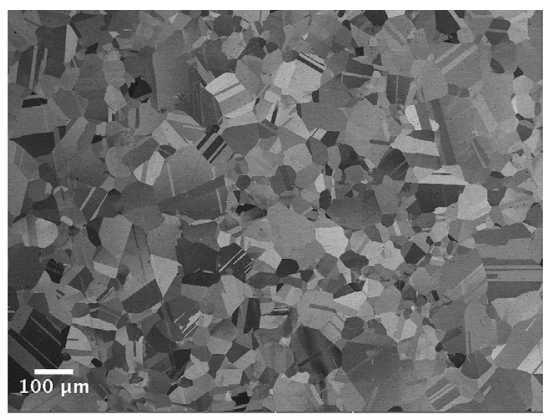

(c)

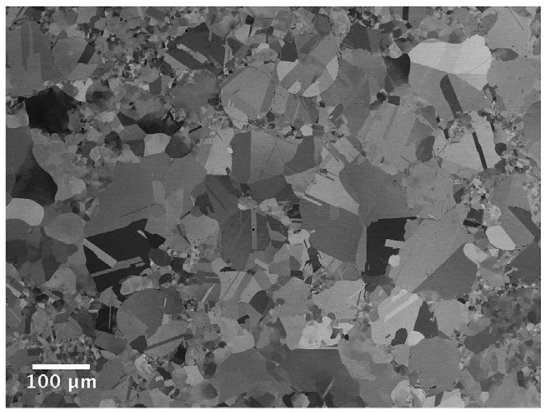

(f)

Fig. 3. BSE images of fully consolidated specimens. (a) specimen HIPed using powder A, (b) to B, (c) to C, (d) to D (e) to E and (f) to F.

shows a representative grain boundary misorientation map of the fully HIPed specimen (using powder D) overlayed with different types of triple junctions. It is seen that most of the triple junctions are of $J_{1}$ type, followed by $J_{0}$ and $J_{2}$, respectively. The distribution of

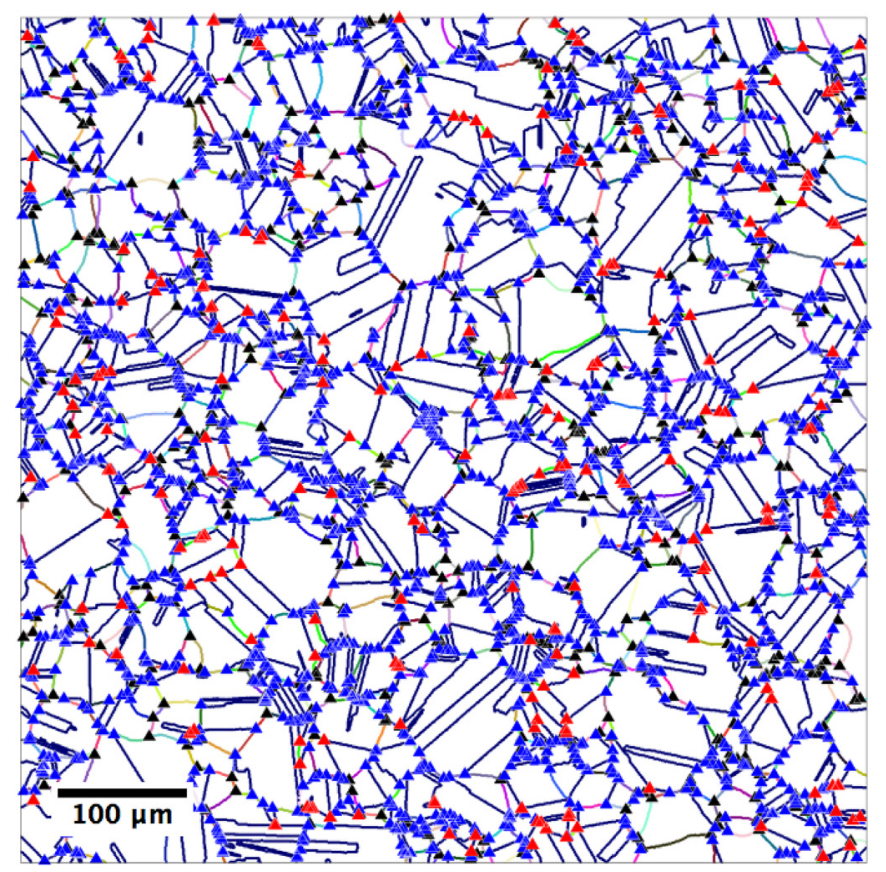

Fig. 4. Representative map showing grain boundary misorientations colour coded according to the legend in Fig. 2b, overlayed with various triple junction types. Here, triple junctions consisting of three non- $\Sigma 3$ boundaries (i.e., $J_{0}$ ) are marked by $\boldsymbol{\Delta}$; one $\Sigma 3$ boundary (i.e., $J_{1}$ ) by $\mathbf{\Delta}$; two $\Sigma 3$ boundaries (i.e., $J_{2}$ ) by $\boldsymbol{\Delta}$ (For interpretation of the references to colour in this figure legend, the reader is referred to the Web version of this article.) types of triple junctions in fully HIPed specimens is tabulated in Table 4 . In all specimens, $J_{1}$ type dominates, followed by $J_{0}$ and $J_{2}$. The statistics also confirm the qualitative observations made from the BSE images (Fig. 3). Specifically, compared to A, B, and F, specimens $C, D$, and $E$ have lower $J_{0}$ type junctions and higher fraction of $J_{1}$ triple junctions. In other words, specimens A, B, and $\mathrm{F}$ have lower number fraction of $\Sigma 3$ boundaries compared to $C, D$ and $\mathrm{E}$; it then follows that they also contain more grains which have not undergone multiple twinning. This further suggests that the observed differences arise from their contrasting powder characteristics (see Fig. 1, and Tables 1-3).

It is emphasized that, owing to the crystallographic constraint at triple junctions [37], the third boundary in a $J_{2}$ type triple junction can either be $\Sigma 9$ or $\Sigma 1$ boundary (i.e., $\Sigma 3^{2}$ and $\Sigma 3^{0}$, respectively), while for the $J_{1}$ type junction, the other two boundaries will be higher order twins (i.e., according to the following rule: $\Sigma 3^{n} \cdot \Sigma 3^{m}=\Sigma 3^{n+m-2 i}$, where $i$ is an integer between 0 and $\left.n\right)[26,28]$. It is also possible that the $J_{0}$ type junctions contain higher order twins (e.g., $\Sigma 9-\Sigma 9-\Sigma 9$ ) and therefore in that respect, the above analysis only gives a rudimentary picture regarding the differences in grain boundary network topology between the specimens. Therefore, in order to identify higher order twins and to better understand the topological differences, we go beyond the quantification of triple junctions associated with $\Sigma 3$ boundaries, and evaluate advanced metrics associated with twin related domains.

Table 4

Triple junction distribution in the fully consolidated specimens.

\begin{tabular}{llll}
\hline Specimen & $J_{0}$ & $J_{1}$ & $J_{2}$ \\
\hline A & $36( \pm 3)$ & $56( \pm 2)$ & $8( \pm 1)$ \\
B & $36( \pm 3)$ & $55( \pm 4)$ & $9( \pm 1)$ \\
C & $31( \pm 2)$ & $62( \pm 2)$ & $7( \pm 1)$ \\
D & $29( \pm 3)$ & $62( \pm 2)$ & $9( \pm 1)$ \\
E & $28( \pm 1)$ & $61( \pm 1)$ & $11( \pm 1)$ \\
F & $36( \pm 3)$ & $56( \pm 3)$ & $8( \pm 1)$ \\
\hline
\end{tabular}


A representative image with TRDs for specimen $\mathrm{E}$ reconstructed using ARPGE is shown in Fig. 5a. Here, the grain boundaries can be identified using the legend shown in Fig. 5b, with the numbers representing $n$ in $\Sigma 3^{n}$. Fig. $5 \mathrm{c}$ is the largest TRD highlighted in Fig. 5a (with an arrow). All grains in this TRD are related by $\Sigma 3^{n}$ misorientations, and are connected by $\Sigma 3$ boundaries; they are shown on a twinning tree for this TRD using a fractal representation (Fig. 5d). All parameters associated with the TRDs are automatically calculated using ARPGE and then averaged.

Fig. $6 \mathrm{a}-\mathrm{f}$ shows the metrics (i.e., Length fraction of $\Sigma 3,\langle T R D\rangle$, $\left\langle N_{g}\right\rangle,\langle L L C\rangle,\langle p\rangle$, and $\left.\langle a\rangle\right)$ quantifying the extent of multiple twinning for the evolutionary microstructural states of all specimens, and very clearly reveal the differences between them. It can be seen that the statistics for all powders HIPed at $950^{\circ} \mathrm{C}$ are very similar, but begin to diverge as the HIPing temperature increases (Fig. 6a-f). This is because, there is incipient plastic deformation of powders at $950^{\circ} \mathrm{C}$, and the corresponding microstructures are similar to those of the as-received powders. Fig. 6a shows the evolution of length fraction of $\Sigma 3$ boundaries as a function of HIPing temperature. While the length fraction in all specimens increases with HIPing temperature, the rate of increase (with temperature and hence, time) is different. In the fully HIPed condition, $\Sigma 3$ boundary length fraction of $C, D$, and $\mathrm{E}$ is similar $(\sim 51 \%)$ and higher than those for $\mathrm{A}$, $\mathrm{B}$, and $\mathrm{F}(\sim 45 \%)$. Specimen $\mathrm{C}$ has the largest $\langle T R D\rangle$ size $(46 \mu \mathrm{m})$, followed by $\mathrm{D}, \mathrm{E}(\sim 31 \mu \mathrm{m})$ then by $\mathrm{F}(23 \mu \mathrm{m})$, and $\mathrm{A}$ and $\mathrm{B}(19 \mu \mathrm{m})$, (Fig: 6b).

The average number of grains in the TRDs (i.e., $\left\langle N_{g}\right\rangle$ ) are lowest for $A, B$, and $F(\sim 3$ per TRD), while those for $C, D$, and $E$ are much higher (3.5, 4, and 4.5, respectively), Fig. $6 \mathrm{c}$. The average length of longest chain $\langle L L C\rangle$ that represents the twinning order in a TRD, is greatest for $\mathrm{E}$ (1.62), followed by $\mathrm{D}(1.55)$ and $\mathrm{C}(1.43)$, and then by $\mathrm{F}$, $A$, and $B(0.98,0.9,0.88$, respectively $)$, Fig. $6 d$. The twins in E, D, and $C$ are more polysynthetic ( $\langle p\rangle$ values are $1.29,1.27,1.26$, respectively) than F, A, and B (1.16,1.15,1.13, respectively), Fig. 6e. The average values for twinning anisotropy $(\langle a\rangle)$ are in the following order: E, C, D (1.83, 1.66, 1.55, respectively), and F, A, and B (1.44, $1.39,1.39$, respectively), Fig. $6 \mathrm{f}$. Fig. $6 \mathrm{a}-\mathrm{f}$ also reveal that the propagation of twin chains is the most during the dwell time of HIPing cycle.

As mentioned previously, powder processed materials usually contain an order of magnitude more oxygen than cast and forged components. Our results show the specimens containing numerous inclusions around $0.5 \mu \mathrm{m}$ in diameter, enriched in manganese and oxygen, although their precise chemical composition was not evaluated (Fig. 7). Most of the inclusions were dislodged during grinding and polishing, leaving "holes" in the matrix, suggesting that they are very weakly bonded to the matrix. This interpretation is consistent with the results from a previous study from our group [18]. Specifically, electropolishing a HIPed 316L specimen resulted in selective removal of the matrix material while the inclusions were retained in the matrix. Moreover, a comparison of microstructures between two specimens with different oxygen contents prepared using mechanical polishing and electropolishing, respectively, revealed that there were more holes (inclusions) in mechanically polished (electropolished) specimens with higher oxygen content, conclusively showing that the holes are in fact inclusions dislodged during mechanical polishing [18].

In order to estimate their fraction in the specimens, the "holes" were treated as oxide inclusions. Their statistical analysis is shown in Table 5. Their density and average size increase with the oxygen

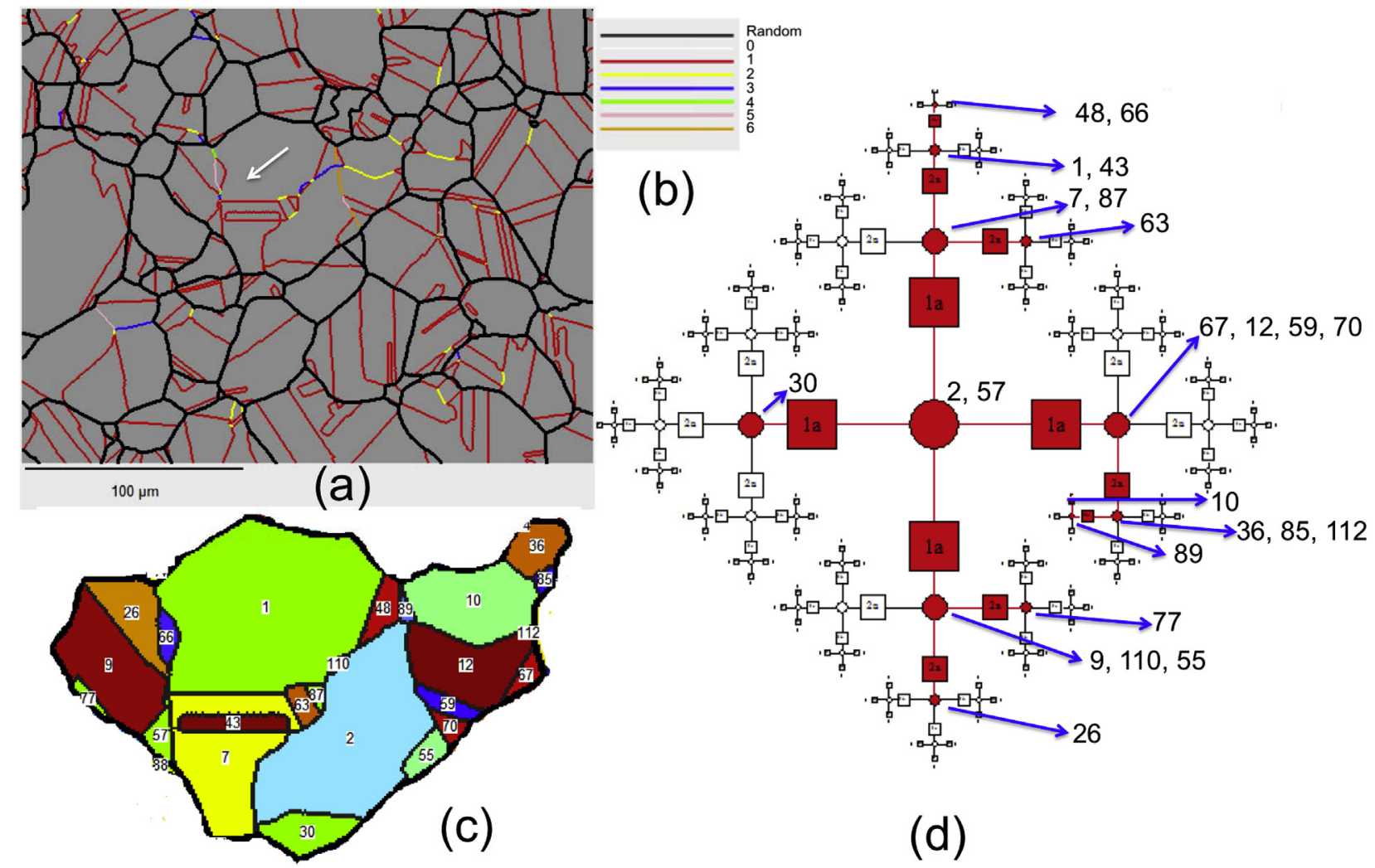

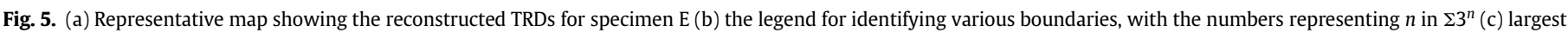

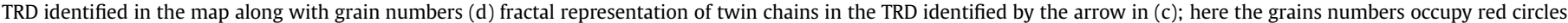

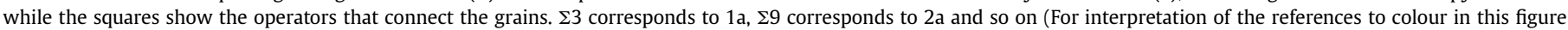
legend, the reader is referred to the Web version of this article.) 


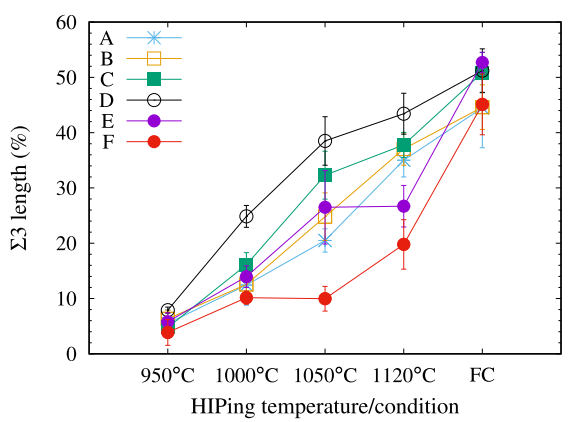

(a)

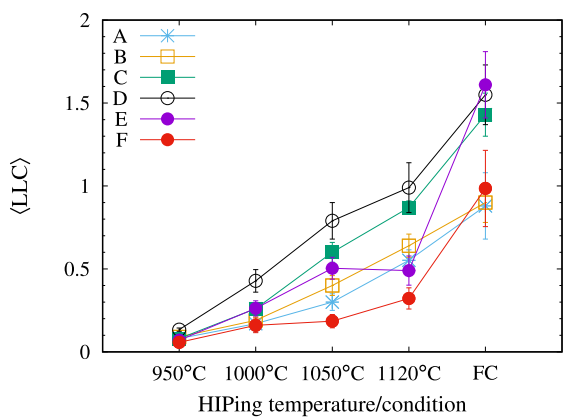

(d)

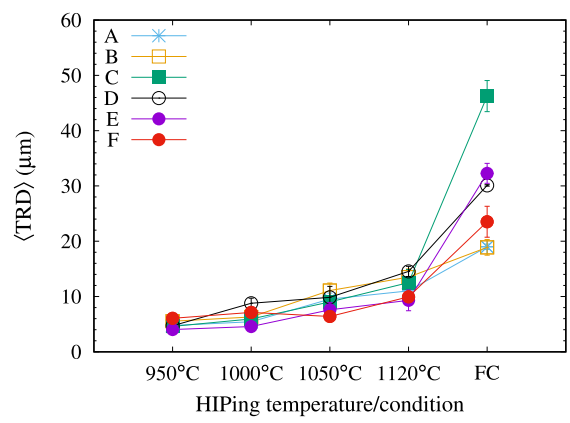

(b)

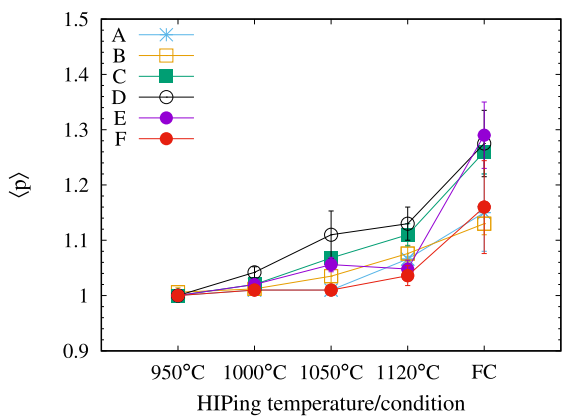

(e)

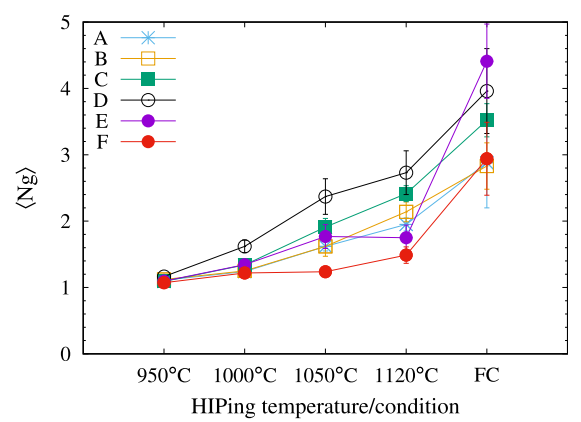

(c)

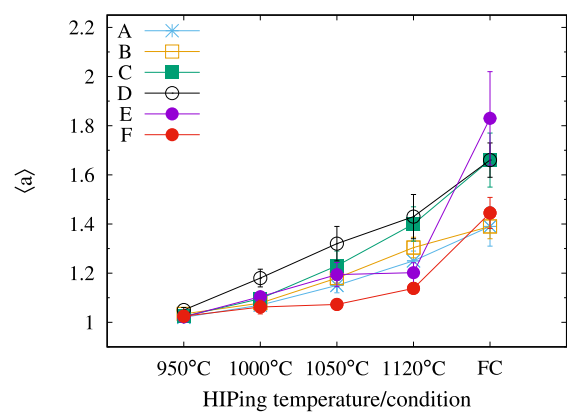

(f)

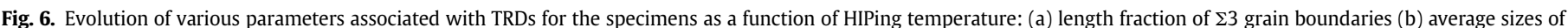

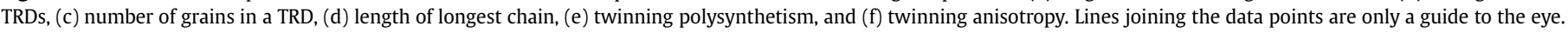
Here FC stands for 'Fully Consolidated'.

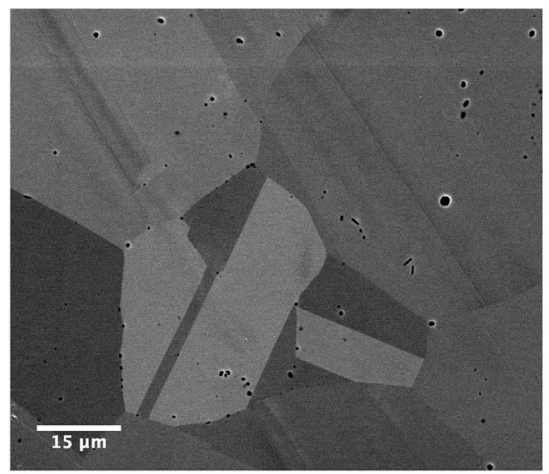

(a)

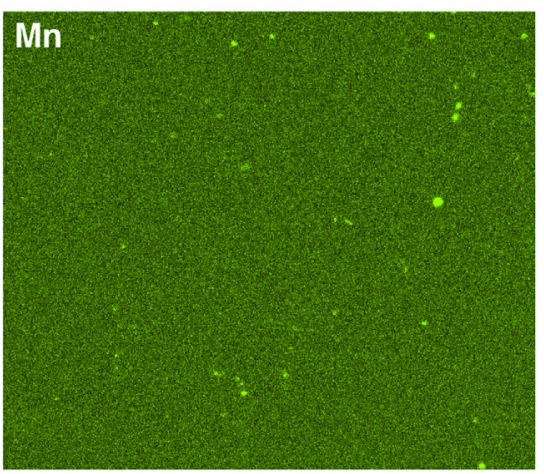

(b)

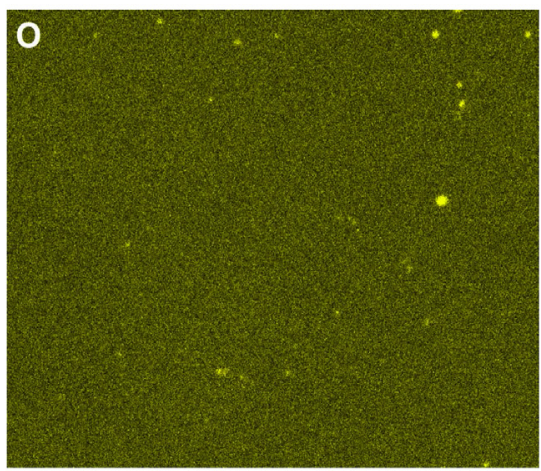

(c)

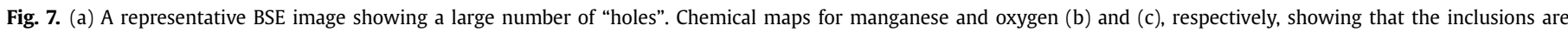
enriched in manganese and oxygen.

Table 5

Inclusion distribution in the fully HIPed microstructure.

\begin{tabular}{|c|c|c|c|c|c|c|}
\hline Powder & A & B & $\mathrm{C}$ & $\mathrm{D}$ & $\mathrm{E}$ & $\mathrm{F}$ \\
\hline Oxygen content & $290 \mathrm{ppm}$ & $110 \mathrm{ppm}$ & 200 ppm & 610 ppm & 220 ppm & 149 ppm \\
\hline Inclusion density $\left(\mathrm{x} 10^{9} / \mathrm{m}^{2}\right)$ & $16( \pm 3)$ & $7.5( \pm 5.5)$ & $8.9( \pm 1)$ & $21.5( \pm 3.6)$ & $12.1( \pm 2.8)$ & $20( \pm 10.7)$ \\
\hline Nearest neighbour distance $(\mu \mathrm{m})$ & $3.64( \pm 0.39)$ & $4.82( \pm 3.9)$ & $4.23( \pm 0.52)$ & $3.23( \pm 0.38)$ & $4.18( \pm 0.58)$ & $2.11( \pm 0.9)$ \\
\hline Average size $(\mu \mathrm{m})$ & $0.46( \pm 0.06)$ & $0.43( \pm 0.09)$ & $0.49( \pm 0.05)$ & $0.5( \pm 0.04)$ & $0.41( \pm 0.07)$ & $0.36( \pm 0.1)$ \\
\hline
\end{tabular}

content in the powders, while the nearest neighbour (NN) distance decreases. Although the oxygen content in specimens $\mathrm{B}$ and $\mathrm{F}$ are very low compared to others ( $110 \mathrm{ppm}$ and $149 \mathrm{ppm}$, respectively), the errors associated with inclusion density and NN distance in them are high. This was observed to be the consequence of their presence along prior particle boundaries (PPBs). Specifically, it was observed that if the PPBs were present in the specimens, inclusions decorated them, and were more closely spaced along them. Moreover, PPBs were only observed in specimens with broad powder size distributions (A, B, and F) and virtually nonexistent in 
specimens $C, D$, and $E$. Interestingly, specimen D which has the highest oxygen content (610 ppm) and a narrow powder size distribution, was homogeneously recrystallized and did not contain any PPBs. In other words, the inclusions were randomly distributed in the bulk. Representative images for specimen $\mathrm{F}(149 \mathrm{ppm})$ and $\mathrm{D}$ $(610 \mathrm{ppm})$ are shown in Fig. 8. PPBs are seen in Fig. 8a which contains fewer annealing twins than in Fig. 8b. The results thus indicate a correlation between (a) powder size distribution and the extent multiple twinning (i.e., recrystallization) (b) oxygen content in the powder and the inclusion density, and (c) spatial distribution of oxide inclusions and recrystallization.

\section{Discussion}

\subsection{Effect of powder size distribution on recrystallization during HIPing}

Depending on the powder size distribution, the extent to which the powder compact is plastically strained during early stages of HIPing varies. Specifically, powders with low tap density experience more strain as they can accommodate more plastic flow than powders with high tap density. In fact, Li and Funkenbusch, in their HIP model, have demonstrated that the deformation of powder in a monosize distribution could be almost twice of that in a bimodal distribution [6]. Since the stored energy increases with the amount of plastic strain experienced, so will the driving force for recrystallization during HIPing. In other words, powder size distribution has a strong effect on the extent of multiple twinning during HIPing. It is pointed out that during high temperature deformation of the powder particles during HIPing, dislocation recovery mechanisms will also be operative, and will affect the microstructural evolution. However, in order to precisely understand them, various high temperature microstructural states need to be preserved by rapid quenching. This is extremely challenging as the cooling rates during the HIPing process, generally, are slow to capture them.

From Fig. 1 and Table 3, it can seen that the powders considered in this study have different size distributions and consequently, varied tap densities (i.e., in the following order: $\mathrm{C}<\mathrm{D}<\mathrm{E}<\mathrm{B}<\mathrm{F}<\mathrm{A}$ ). Therefore, the metrics quantifying multiple twinning can be expected to follow a similar trend, although it must be noted that locally, powders experience strains that will be different from the macroscopic strain due to the shrinkage of canisters during HIPing. In other words, while the differences in tap density/powder size distribution give an indication of the relative strains, the development of multiple twinned microstructure depends on the energy stored locally. More specifically, it is analogous to the dependence of applied strain on the differences in the extent of multiple twinning observed in the grain boundary engineering studies of low-medium SFE materials (e.g., Refs. [38-40]).

From Table 4, which shows the distribution of various triple junction types, it can be seen that $J_{1}$ type junctions are higher in specimens $\mathrm{C}, \mathrm{D}$, and $\mathrm{E}$ compared to $\mathrm{A}, \mathrm{B}$, and $\mathrm{F}$. In other words, the number of annealing twins is higher in $C, D$, and $E$ than in $A, B, F$. The average number of grains, $\left\langle N_{g}\right\rangle$, within TRDs is highest in $\mathrm{E}$, followed by D and C, while they are lowest for A, B, and F. It suggests that the twin chains in specimens $C, D, E$ have propagated more than those in A, B, and F. This is further confirmed if we compare $\langle L L C\rangle,\langle p\rangle$, and $\langle a\rangle$. It is seen that these parameters are nicely grouped for C, D, E (a shade higher), and A, B, F (comparitively lower), clearly revealing that broad powder size distributions, owing to higher tap densities (and consequently, experiencing lower plastic strains during HIPing), have lower driving force for recrystallization compared to powders with lower tap densities (Table 3). In summary, a narrow powder size distribution with low tap density produces a homogeneously recrystallized microstructure. Such a microstructure is beneficial from the point of view of enhanced resistance towards material degradation mechanisms that propagate intergranularly (e.g., stress corrosion cracking).

\subsection{Mechanism of formation of oxide inclusions}

As previously pointed out, during the early stages of HIPing, plastic deformation of powders is initiated at their contact points with other powders. The temperature and pressure at which the deformation starts and proceeds depends on the elevated temperature strength of the material and the powder size distribution. Hedberg et al. have shown that gas atomised 316L powder surface consists of a homogeneous layer $(<10 \mathrm{~nm})$ of iron/manganese/ chromium oxide [41]. Because of the presence of a continuous oxide film, complete bonding between powders during their incipient deformation at elevated temperature (i.e., at $\sim 950^{\circ} \mathrm{C}$ in this study) cannot occur. The oxide layer is disrupted during HIPing and metal to metal contacts are established. In order to reduce the interfacial energy between the metal and oxide layer, the disrupted oxide layer coalesces to form inclusions $(\sim 0.2 \mu \mathrm{m})$ at the interparticle boundaries, Fig. 9a.

With increasing temperature, powders experience larger strains and simultaneously begin to recrystallize, Fig. 9b, where the presence of annealing twins can be seen. However, the inclusions are still present at the inter-particle boundaries. At $1050^{\circ} \mathrm{C}$, depending on the local plastic strain experienced by the powders, the recrystallized grains begin to grow past the inter-particle boundaries with the inclusions still arranged on them, Fig. 9c. However, after the full HIP cycle (i.e., HIPed at $1160{ }^{\circ} \mathrm{C}, 103 \mathrm{MPa}, 4 \mathrm{~h}$ dwell

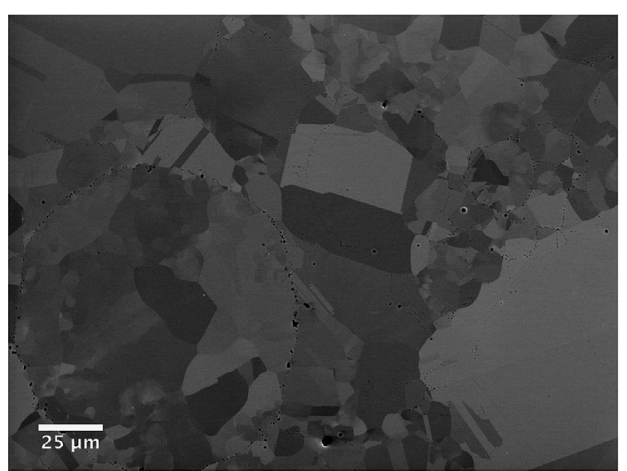

(a)

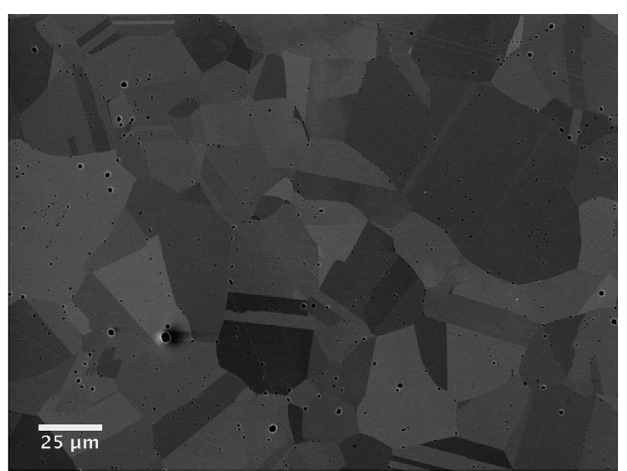

(b)

Fig. 8. Secondary electron showing the effect of recrystalization on inclusion distribution in fully HIPed specimen of powder F (a) and D (b). 


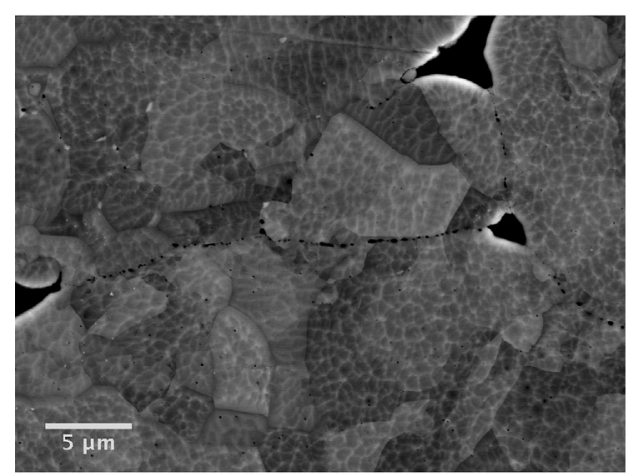

(a) $950^{\circ} \mathrm{C}$

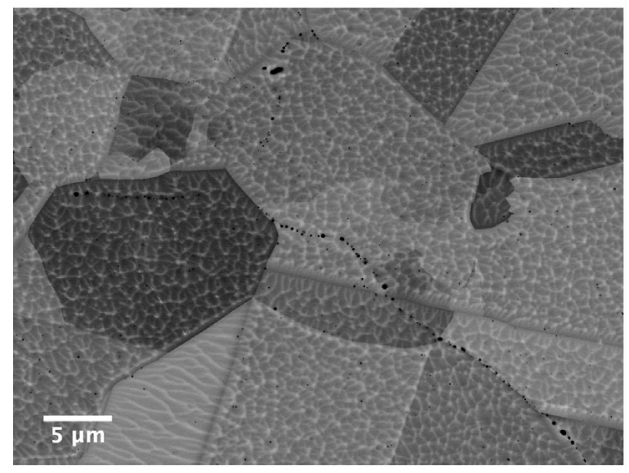

(c) $1050^{\circ} \mathrm{C}$

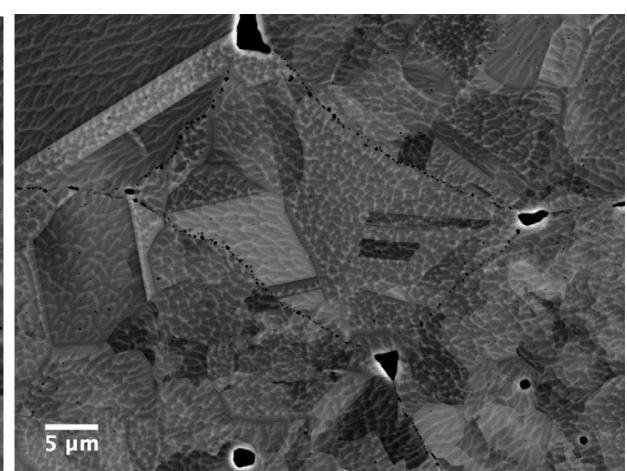

(b) $1000^{\circ} \mathrm{C}$

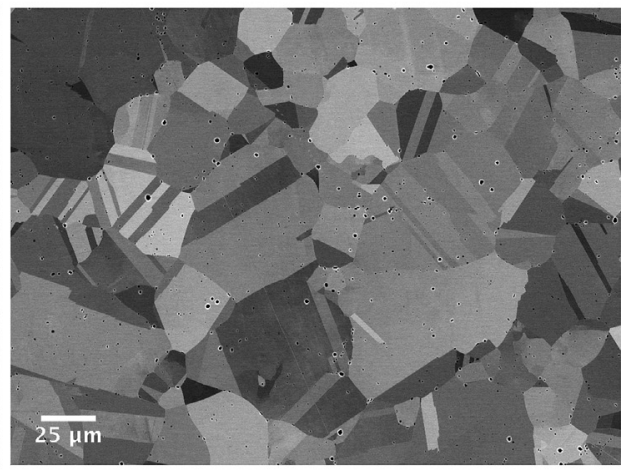

(d) Fully HIPed

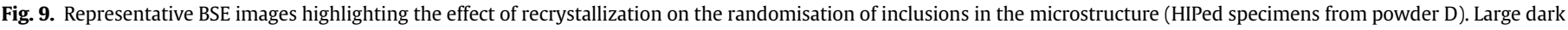
regions between the powders in (a) and (b) correspond to the porosity resulting from partial consolidation.

time), the inclusions are distributed in the bulk, depending on the homogeneity of recrystallization, albeit there are few regions where they are situated at the grain boundaries (Fig. 9d), indicating that inclusions are dragged by moving grain boundaries during recrystallization. Moreover, their average size has also increased to around $0.5 \mu \mathrm{m}$ (refer Table 5), further suggesting that the coarsening of inclusions is diffusion and/or coalescence induced. We note that the growth of oxide inclusions during recrystallization has previously been reported for $\mathrm{Ni}-\mathrm{Cr}$ and $\mathrm{Cu}-\mathrm{Si}$ alloys, respectively $[42,43]$. Once nucleated, the oxide inclusions must be quite stable at HIPing temperatures (i.e., $1160{ }^{\circ} \mathrm{C}$ ). This is because, if they were to be in solid solution, there is no a priori reason for their renucleation exactly at PPBs during the cooling step of HIPing process. We further note the presence of sub-micron sized cellular microstructure in the partially HIPed specimens (evident in Fig. $9 a-c)$, which is the consequence of rapid solidification of the melt during gas atomization process (i.e., powder production process). However, the microstructure homogenizes during the "dwell time" of the HIPing cycle.

\subsubsection{Effect of oxygen content on recrystallization}

The powders considered in the present study had oxygen content ranging from $110 \mathrm{ppm}$ (powder B) to $610 \mathrm{ppm}$ (powder D), Table 2. We now compare the metrics for specimens B and D (in other words, the lowest and highest oxygen content specimens, respectively) to specifically understand the effect of oxygen content in the powder on recrystallization during HIPing. Firstly, the microstructures qualitatively reveal that specimen $B$ has recrystallized inhomogeneously while specimen $\mathrm{D}$ has a more homogeneous microstructure. The triple junction distributions (Table 4) quantitatively indicate a higher fraction of $\Sigma 3$ boundaries in D compared to $\mathrm{B}$ (fraction of $J_{0}$ is lower while $J_{1}$ is higher in $\mathrm{D}$ ). In addition, the advanced metrics related to TRDs (i.e., $\langle T R D\rangle,\left\langle N_{g}\right\rangle,\langle L L C\rangle,\langle p\rangle$, and $\langle a\rangle)$ show that the extent of multiple twinning is much larger in specimen $\mathrm{D}$ than in $\mathrm{B}$, clearly indicating that the oxygen content has no effect on recrystallization. On the other hand, the powder size distribution of B is much broader than $\mathrm{D}$ (tap densities of $5.5 \mathrm{~g} \mathrm{~cm}^{-3}$ and $5.2 \mathrm{~g} \mathrm{~cm}^{-3}$, respectively) due to which the plastic strain (and hence stored energy) in the compact of powder B during HIPing will be lower than powder $\mathrm{D}$. This in turn results in larger driving force for recrystallization in D and hence, better twinning stastistics than in B.

Rao et al. studied the effect of oxygen content on the microstructure of powder HIPed Inconel 718 and concluded that the effect of oxygen content in the powders is to retard recrystallization [12]. Specifically, using three powders with different oxygen contents (275 ppm, $180 \mathrm{ppm}$, and $140 \mathrm{ppm}$ ) they observed (qualitatively, from the micrographs) that the extent of recrystallization decreased with an increase in oxygen content, although the effect of powder size distribution was ignored in their analysis (refer Figs. 2c and 3c in Ref. [12]). More specifically, the tap density was highest for the powder with $275 \mathrm{ppm}$ oxygen (broad size distribution) and lowest for $140 \mathrm{ppm}$ (narrow size distribution), which clearly suggests that the driving force for recrystallization for the powder with lowest tap density will be greater than that for highest tap density. We believe that the results of Rao et al. tacitly demonstrated the dependence of powder size distribution on recrystallization, but were interpreted otherwise. In summary, our results suggest that the oxygen content in the powders has no effect on the degree of plastic strain experienced during initial stages of HIPing, although in order to prove it conclusively, powders with same particle size distribution but different oxygen content need to 
be HIPed. For a particular size distribution of powders, the oxygen content, for example, can be increased by heat treating them under controlled conditions.

\subsubsection{Spatial distribution of oxide inclusions during consolidation and the effect of recrystallization}

While powder HIPed components can be manufactured in near net shape, one of the challenges in using them in high integrity structural applications is their lower toughness values (attributed to the presence of oxide inclusions) compared to cast and forged ones, although it is emphasized that the toughness values in many cases are still above the ones stipulated in ASME codes. See for e.g. Refs. [17,18], for the case of powder HIPed 316L and 304L. The size, volume fraction, coherency with the matrix, and spatial distribution of inclusions have a strong effect on the material toughness. It is emphasized that powder particles, regardless of their size, contain an oxide layer on their surface; the amount of oxygen depends on their size, gas atomization process, and storage conditions. Presence of PPBs, and therefore, inclusions decorating them, indicates that the corresponding particles have not plastically deformed during HIPing process. Fig. 8 shows the effect of recrystallization on the spatial distribution of inclusions. Specifically, Fig. 8a, which corresponds to powder F (highest tap density and very low oxygen content), reveals a particular region where the inclusions are decorated at PPBs while Fig. 8b, which corresponds to powder D (significantly lower tap density and highest oxygen content), shows a random distribution of the inclusions. The triple junction distribution and twinning statistics reveal that the scale and homogeneity of recrystallization in specimen $\mathrm{D}$ is much better than specimen $\mathrm{F}$, clearly demonstrating that recrystallization strongly affects the spatial distribution of the inclusions.

During powder HIPing, a general view regarding the effect of inclusions at PPBs is that they act as pinning centres for moving grain boundaries [12,19,44-46]. Indeed, one of the ways suggested to promote the movement of grain boundaries past them is to perform HIPing at temperatures where the driving force for the boundary movement is greater than the pinning force of the inclusions at PPBs, although it is recognized that it results in increased grain size $[13,19]$. However, pushing of particles by moving grain boundaries during solid state phase transformations (e.g., recrystallization, allotropic transformation), and diffusional movement of inert particles have previously been reported [42,43,47-52]. These studies focused on the effect of recrystallization in a material with homogeneous dispersion of inclusions. However, in powder HIPing, the opposite holds; i.e., the inclusions nucleate at inter-particle boundaries (or PPBs) where the contact stresses are the highest (provided there is room for plastic flow), and are then pushed away from PPBs as a result of recrystallization during the dwell time of the HIPing cycle.

While it is known that, in low-medium SFE materials, recrystallization occurs during HIPing, previous studies have not explicitly correlated the spatial distribution of inclusions to multiple twinning. In fact, results from this study have clearly demonstrated that, although the inclusions nucleate at the inter-particle boundaries, their spatial distribution after the HIPing cycle (i.e., after complete consolidation) is strongly affected by the extent of recrystallization, which is governed by the powder size distribution. Therefore, in a much broader context, the naturally occurring mechanisms of phase transformation and related phenomena during powder processing of certain alloy systems (e.g., recrystallization in low-medium SFE materials, transformation mismatch plasticity in titanium alloys and martensitic/bainitic steels; see for e.g., Refs. [53,54]) can be used for optimizing the manufacturing process and controlling the microstructural evolution.

\subsection{Implications of the present study for powder-HIPed components with low-medium SFE}

One of the main results originating from the present study is that, the homogeneity of recrystallization during HIPing is governed by the powder size distribution and not by the amount of oxygen content. More importantly, the spatial distribution of oxide inclusions and their volume fraction are governed by the extent of recrystallization and the amount of oxygen content in the powders, respectively. We believe that these observations are very important and are of high value, and can be used to potentially manufacture near net shape components for critical applications only by powder-HIPing. As an example, we identify its applicability to powder processed nickel-base superalloys.

Highly alloyed nickel-base superalloys which are of interest for demanding environments, are processed using powder metallurgy techniques to avoid casting induced segregation of alloying elements. However, presence of PPBs in the as-HIPed condition has been a perennial problem, and has necessitated post-HIP thermomechanical processing of these alloys, which not only increases the manufacturing cost, but also restricts the efficient use of HIPing as a process for manufacturing near net shape components. Specifically, processing involves canning of gas-atomised powders and HIPing, followed by hot extrusion and/or isothermal forging [20,21]. PostHIP thermomechanical processing is performed in order to "break" the PPB network present in the as-HIPed products (i.e., via recrystallization). Hot-working of these alloys after HIPing has been shown to be effective not only in enhancing the fraction of $\Sigma 3$ boundaries, but also in breaking the PPB networks $[22,55,56]$, clearly suggesting a direct correlation between the extent and homogeneity of recrystallization and the absence of PPBs.

In our study on powder HIPed 316L, by considering different powder size distributions, and by just HIPing, we clearly demonstrated a correlation between the extent and homogeneity of recrystallization, and the absence of PPBs (Fig. 3, Table 4, Figs. 6, and Fig. 8). More specifically, a narrow powder size distribution has produced a more homogeneously recrystallized microstructure free from PPBs than the broadly distributed ones. While a broad powder size distribution (in other words, distribution with high tap density) provides better control over the shape change of the component after HIPing, ${ }^{2}$ modelling can be used to predict the shape change so that the initial canister can be designed in order to obtain near net shape after HIPing even with narrow powder size distribution. The benefit of uniform recrystallization during HIPing is two-fold. First, the increased fraction of $\Sigma 3$ grain boundaries due to multiple twinning during HIPing can improve high cycle fatigue crack propagation behaviour [57]. Second, the inclusions that nucleate at inter-particle boundaries will more likely be dispersed homogeneously after HIPing (because of the absence of PPBs), potentially improving the ductility, although it must be noted that any improvement in ductility is dependent on the volume fraction of inclusions.

While it can be argued that the propensity for twinning depends on the SFE of the material, and that there would be differences in microstructural topology between $316 \mathrm{~L}$ and $\mathrm{Ni}$-base superalloys, we emphasize that hot working has a strong effect on grain boundary network topology, and we also underscore that HIPing is essentially a thermomechanical process. For example, within the context of twinning induced grain boundary engineering in castings, the effect of process variables (e.g., strain, annealing

\footnotetext{
2 During HIPing, shrinkage of the canister is more uniform for a broad powder size distribution compared to a narrow size because of the lesser extent of plastic
} flow offered. 
temperature and time, or strain rate and hot deformation temperature) on grain boundary connectivity can be inferred by referring to Ref. [58] for a general overview and [59-61] specifically for Nibase superalloys. In the case of powder HIPing, the process variables are powder size distribution, temperature, pressure, and time.

The effectiveness of HIPing as a thermomechanical process can therefore be taken advantage of, to reduce/potentially eliminate the problem of PPBs by proper choice of powder size distribution, chemistry, and HIPing parameters. More specifically, the inclusion density can be reduced by using powder with low oxygen content while their spatial distribution can be randomized by optimizing the HIP process variables to promote homogeneous recrystallization. That is, in addition to using narrow powder size distribution, the stored energy in the deformed powders can be tuned by adjusting the way in which pressure and temperature are applied in order to promote multiple twinning and eliminate PPBs during HIPing. While the results in the present study were obtained on specimens with relatively simple geometry, getting a homogeneous microstructure in a large complex HIPed part, due to potential variations in strain distribution, could be challenging, and needs to be demonstrated. In this regard, additional studies focusing on effect of powder characteristics and HIP process variables on the spatial variations in microstructural features in large powder-HIPed components are needed.

\section{Conclusions}

The aim of the present study was to understand the effect of powder characteristics and oxygen content on the microstructural evolution during hot isostatic pressing of 316L austenitic steel. The main findings are summarized as follows:

- The principal mechanism by which the microstructure evolves during HIPing is recrystallization, the homogeneity of which, is strongly dependent on the powder size distribution.

- Fully HIPed specimens with narrow powder size distribution (i.e., lower tap density) were more homogeneously recrystallized than the ones produced using broad size distribution (i.e., higher tap density). Prior particle boundaries (PPBs) were more frequently observed in specimens HIPed with broad powder size distribution than the ones HIPed with narrow distribution.

- The density of oxide inclusions in the fully HIPed specimens increased with the oxygen content in the powders, with their spatial distribution strongly dependent on the homogeneity of recrystallization.

- The oxide inclusions rich in manganese nucleated on interparticle boundaries (in other words, PPBs) during early stages of HIPing, and depending on whether or not there was sufficient stored energy, were pushed by the moving grain boundaries during recrystallization.

Finally, as demonstrated here, homogeneous recrystallization during HIPing results in random distribution of oxide inclusions and a microstructure free from PPBs. This is highly relevant from the point of view of manufacturing PPB free near net shape components for critical applications, since it would eliminate the need for post-HIP thermomechanical processing (in order to break the PPBs) and reduce manufacturing costs.

\section{Acknowledgements}

The authors would like to thank the EPSRC for funding through New Nuclear Manufacturing (NNUMAN): EP/J021172/1, and Manufacture using Advanced Powder Processes (MAPP): EP/ P006566/1. Rolls-Royce is also thanked for supplying the $316 \mathrm{~L}$ forging for this study.

\section{References}

[1] H.V. Atkinson, S. Davies, Fundamental aspects of hot isostatic pressing: an overview, Metall. Mater. Trans. 31A (2000) 2981-3000.

[2] D.S. Wilkinson, M.F. Ashby, Pressure sintering by power law creep, Acta Metall. 23 (11) (1975) 1277-1285.

[3] E. Arzt, The influence of an increasing particle coordination on the densification of spherical powders, Acta Metall. 30 (10) (1982) 1883-1890.

[4] A.S. Helle, K.E. Easterling, M.F. Ashby, Hot-isostatic pressing diagrams: New developments, Acta Metall. 33 (12) (1985) 2163-2174.

[5] S.V. Nair, J.K. Tien, Densification mechanism maps for hot isostatic pressing (HIP) of unequal sized particles, Metallurgical Transactions A 18 (1) (1987) 97-107.

[6] E.K.H. Li, P.D. Funkenbusch, Hot isostatic pressing (hip) of powder mixtures and composites: packing, densification, and microstructural effects, Metallurgical Transactions A 24 (6) (1993) 1345-1354.

[7] D.P. Delo, H.R. Piehler, Early stage consolidation mechanisms during hot isostatic pressing of Ti-6Al-4V powder compacts, Acta Mater. 47 (9) (1999) 2841-2852.

[8] W.A. Kaysser, M. Aslan, E. Arzt, M. Mitkov, G. Petzow, Microstructural development and densification during hipping of ceramics and metals, Powder Metall. 31 (1) (1988) 63-69.

[9] R.N. Wright, R.L. Williamson, J.R. Knibloe, Modelling of hipping consolidation applied to $\mathrm{Ni}_{3} \mathrm{Al}$ powders, Powder Metall. 33 (3) (1990) 253-259.

[10] H.R. Piehler, D.P. Delo, Physical modeling of powder consolidation processes, Prog. Mater. Sci. $42(1-4)(1997)$ 263-276.

[11] S. Irukuvarghula, H. Hassanin, C. Cayron, M.M. Attallah, D. Stewart, M. Preuss, Evolution of grain boundary network topology in 316L austenitic stainless steel during powder hot isostatic pressing, Acta Mater. 133 (2017) 269-281.

[12] G.A. Rao, M. Kumar, M. Srinivas, D.S. Sarma, Effect of oxygen content of powder on microstructure and mechanical properties of hot isostatically pressed superalloy Inconel 718, Mater. Sci. Eng., A 435-436 (2006) 84-99.

[13] L. Chang, W. Sun, Y. Cui, R. Yang, Influences of hot-isostatic-pressing temperature on microstructure, tensile properties and tensile fracture mode of Inconel 718 powder compact, Mater. Sci. Eng., A 599 (2014) 186-195.

[14] C.F. Tipper, The fracture of metals, Metallurgia 39 (1949) 133-137.

[15] K.E. Puttick, Ductile fracture in metals, Phil. Mag. 4 (44) (1959) 964-969.

[16] A. Pineau, A.A. Benzerga, T. Pardoen, Failure of metals I: brittle and ductile fracture, Acta Mater. 107 (2016) 424-483.

[17] A.J. Cooper, N.I. Cooper, A. Bell, J. Dhers, A.H. Sherry, A microstructural study on the observed differences in charpy impact behavior between hot isostatically pressed and forged 304L and 316L austenitic stainless steel, Metall. Mater. Trans. 46 (11) (2015) 5126-5138.

[18] A.J. Cooper, N.I. Cooper, J. Dhers, A.H. Sherry, Effect of oxygen content upon the microstructural and mechanical properties of type 316L austenitic stainless steel manufactured by hot isostatic pressing, Metall. Mater. Trans. 47 (9) (2016) 4467-4475.

[19] C.L. Qiu, M.M. Attallah, X.H. Wu, P. Andrews, Influence of hot isostatic pressing temperature on microstructure and tensile properties of a nickel-based superalloy powder, Mater. Sci. Eng., A 564 (2013) 176-185.

[20] X. Pierron, A. Banik, G.E. Maurer, J. Lemsky, D.U. Furrer, S. Jain, Sub-solidus HIP process for P/M superalloy conventional billet conversion, Superalloys (2000) 425-433.

[21] M.C. Hardy, B. Zirbel, G. Shen, R. Shankar, Developing damage tolerance and creep resistance in a high strength nickel alloy for disc applications, Superalloys (2004) 83-90, 2004.

[22] G.A. Rao, M. Srinivas, D.S. Sarma, Effect of thermomechanical working on the microstructure and mechanical properties of hot isostatically pressed superalloy Inconel 718, Mater. Sci. Eng., A 383 (2) (2004) 201-212.

[23] S.L. Semiatin, K.E. McClary, A.D. Rollett, C.G. Roberts, E.J. Payton, F. Zhang, T.P. Gabb, Microstructure evolution during supersolvus heat treatment of a powder metallurgy nickel-base superalloy, Metall. Mater. Trans. 43 (5) (May 2012) 1649-1661.

[24] S.L. Semiatin, K.E. McClary, A.D. Rollett, C.G. Roberts, E.J. Payton, F. Zhang T.P. Gabb, Plastic flow and microstructure evolution during thermomechanical processing of a PM nickel-base superalloy, Metall. Mater. Trans. 44 (6) (Jun 2013) 2778-2798.

[25] V.Y. Gertsman, C.H. Henager, Grain boundary junctions in microstructure generated by multiple twinning, Interface Sci. 11 (4) (2003) 403-415.

[26] B.W. Reed, R.W. Minich, R.E. Rudd, M. Kumar, The structure of the cubic coincident site lattice rotation group, Acta Crystallogr. A 60 (3) (May 2004) $263-277$.

[27] W.R. Bryan, M. Kumar, Mathematical methods for analyzing highly-twinned grain boundary networks, Scripta Mater. 54 (6) (2006) 1029-1033. Viewpoint set no. 40: Grain boundary engineering.

[28] C. Cayron, Multiple twinning in cubic crystals: geometric/algebraic study and its application for the identification of the $\sum 3^{n}$ grain boundaries, Acta Crystallogr. A: Foundations of Crystallography 63 (1) (2007) 11-29.

[29] C. Cayron, Quantification of multiple twinning in face centred cubic materials, Acta Mater. 59 (1) (2011) 252-262.

[30] B.W. Reed, M. Kumar, R.W. Minich, R.E. Rudd, Fracture roughness scaling and its correlation with grain boundary network structure, Acta Mater. 56 (13) 
(2008) 3278-3289.

[31] C.M. Barr, S. Thomas, J.L. Hart, W. Harlow, E. Anber, M.L. Taheri, Tracking the evolution of intergranular corrosion through twin-related domains in grain boundary networks, Npj Mater. Degrad. 2 (1) (2018) 14.

[32] R. Hielscher, H. Schaeben, A novel pole figure inversion method: specification of the MTEX algorithm, J. Appl. Crystallogr. 41 (6) (Dec 2008) 1024-1037.

[33] C. Cayron, ARPGE: a computer program to automatically reconstruct the parent grains from electron backscatter diffraction data, J. Appl. Crystallogr. 40 (6) (Dec 2007) 1183-1188.

[34] C.A. Schneider, W.S. Rasband, K.W. Eliceiri, NIH Image to ImageJ: 25 years of image analysis, Nat. Methods 9 (7) (2012) 671-675.

[35] S. Patala, C.A. Schuh, A continuous and one-to-one coloring scheme for misorientations, Acta Mater. 59 (2) (2011) 554-562.

[36] S. Patala, J.K. Mason, C.A. Schuh, Improved representations of misorientation information for grain boundary science and engineering, Prog. Mater. Sci. 57 (8) (2012) 1383-1425.

[37] K. Miyazawa, Y. Iwasaki, K. Ito, Y. Ishida, Combination rule of $\sum$ values at triple junctions in cubic polycrystals, Acta Crystallogr. A: Foundations of Crystallography 52 (6) (1996) 787-796.

[38] M. Shimada, H. Kokawa, Z.J. Wang, Y.S. Sato, I. Karibe, Optimization of grain boundary character distribution for intergranular corrosion resistant 304 stainless steel by twin- induced grain boundary engineering, Acta Mater. 50 (2002) 2331-2341.

[39] C.A. Schuh, M. Kumar, W.E. King, Analysis of grain boundary networks and their evolution during grain boundary engineering, Acta Mater. 51 (2003) 687-700.

[40] M. Michiuchi, H. Kokawa, ZJ. Wang, Y.S. Sato, K. Sakai, Twin-induced grain boundary engineering for 316 austenitic stainless steel, Acta Mater. 54 (2006) 5179-5184.

41] Y. Hedberg, M. Norell, J. Hedberg, P. Szakalos, P. Linhardt, I.O. Wallinder, Surface characterisation of fine inert gas and water atomised stainless steel 316L powders: formation of thermodynamically unstable surface oxide phases, Powder Metall. 56 (2) (2013) 158-163.

[42] V.N. Antsiferov, R.R. Yablonovskaya, Growth of dispersed oxide inclusions in $80 \% \mathrm{Ni}-20 \% \mathrm{Cr}$ alloys during annealing, Sov. Powder Metall. Met. Ceram. 10 (6) (Jun 1971) 482-484.

[43] A.V. Serebryakov, T.M. Redkova, V.I. Lobanov, On recrystallization of dispersion-hardened alloys, Phys. Status Solidi 14 (1) (1972) 77-81.

[44] D.L. Williams, Hot isostatically pressed alloy APK1, a nickel-base superalloy, Powder Metall. 20 (2) (1977) 84-89.

[45] D. Furrer, H. Fecht, Ni-based superalloys for turbine discs, J. Met. 51 (1) (1999) $14-17$.
[46] G.A. Rao, M. Kumar, M. Srinivas, D.S. Sarma, Effect of standard heat treatment on the microstructure and mechanical properties of hot isostatically pressed superalloy inconel 718, Mater. Sci. Eng., A 355 (1-2) (2003) 114-125.

[47] M.F. Ashby, I.G. Palmer, The dragging of solid particles through metals by grain boundaries, Acta Metall. 15 (2) (1967) 420-423.

[48] M.F. Ashby, R.M.A. Gentamore, The dragging of small oxide particles by migrating grain boundaries in copper, Acta Metall. 16 (9) (1968) 1081-1092.

[49] E.F. Koch, K.T. Aust, The movement of solid particles in a solid, Acta Metall. 15 (2) (1967) 405-409.

[50] G.I. Kokhanchik, A.V. Serebryakov, Yu A. Shiyanov, Diffusion movement of particles in solids, Phys. Status Solidi 23 (1) (1974) 99-103.

[51] G. Gottstein, L.S. Shvindlerman, Theory of grain boundary motion in the presence of mobile particles, Acta Metall. Mater, 41 (11) (1993) 3267-3275.

[52] R.K. Shelton, D.C. Dunand, Computer modeling of particle pushing and clustering during matrix crystallization, Acta Mater. 44 (11) (1996) 4571-4585.

[53] Y. Oshida, An application of superplasticity to powder metallurgy, J. Jap. Soc. of Powder Metall. 22 (5) (1975) 147-153.

[54] C. Schuh, P. Noel, D.C. Dunand, Enhanced densification of metal powders by transformation-mismatch plasticity, Acta Mater. 48 (8) (2000) 1639-1653.

[55] M. Detrois, J. Rotella, R.L. Goetz, R.C. Helmink, S. Tin, Grain boundary engineering of powder processed Ni-base superalloy RR1000: influence of the deformation parameters, Mater. Sci. Eng., A 627 (2015) 95-105.

[56] R.C. Buckingham, C. Argyrakis, M.C. Hardy, S. Birosca, The effect of strain distribution on microstructural developments during forging in a newly developed nickel base superalloy, Mater. Sci. Eng., A 654 (2016) 317-328.

[57] Y. Gao, R.O. Ritchie, M. Kumar, R.K. Nalla, High-cycle fatigue of nickel-based superalloy ME3 at ambient and elevated temperatures: role of grainboundary engineering, Metall. Mater. Trans. 36 (12) (2005) 3325-3333.

[58] V. Randle, Twinning-related grain boundary engineering, Acta Mater. 52 (14) (2004) 4067-4081.

[59] M. Detrois, R.L. Goetz, R.C. Helmink, S. Tin, Modeling the effect of thermalmechanical processing parameters on the density and length fraction of twin boundaries in Ni-base superalloy RR1000, Mater. Sci. Eng., A 647 (2015) $157-162$.

[60] M. Detrois, J. McCarley, S. Antonov, R.C. Helmink, R.L. Goetz, S. Tin, Comparative study of high-temperature grain boundary engineering of two powderprocessed low stacking-fault energy Ni-base superalloys, Mater. A. T. High. Temp. 33 (4-5) (2016) 310-317.

[61] J. McCarley, R. Helmink, R. Goetz, S. Tin, Grain boundary engineering of a low stacking fault energy Ni-based superalloy, Metall. Mater. Trans. 48 (4) (2017) 1666-1677. 